\title{
Numerical Study of Wetting Transitions on Biomimetic Surfaces Using a Lattice Boltzmann Approach with Large Density Ratio
}

\author{
Wei Gong ${ }^{1}$, Yuying Yan $^{1 *, 2}$, Sheng Chen ${ }^{1}$, Donald Giddings ${ }^{1}$ \\ ${ }^{1}$ Fluids \& Thermal Engineering Research Group, Faculty of Engineering, University of Nottingham, \\ University Park, Nottingham, NG7 2RD, UK \\ ${ }^{2}$ Center for Fluids \& Thermal Engineering, University of Nottingham Ningbo, China
}

*corresponding author: yuying.yan@nottingham.ac.uk

\begin{abstract}
The hydrophobicity of natural surfaces have drawn much attention of scientific communities in recent years. By mimicking natural surfaces, the manufactured biomimetic hydrophobic surfaces have been widely applied to green technologies such as self-cleaning surfaces. Although the theories for wetting and hydrophobicity have been developed, the mechanism of wetting transitions between heterogeneous wetting state and homogeneous wetting state is still not fully clarified. As understanding of wetting transitions is crucial for manufacturing a biomimetic superhydrophobic surface, more fundamental discussions in this area should be carried out. In the present work the wetting transitions are numerically studied using a phase field lattice Boltzmann approach with large density ratio, which should be helpful in understanding the mechanism of wetting transitions. The dynamic wetting transition processes between Cassie-Baxter state and Wenzel state are presented, and the energy barrier and the gravity effect on transition are discussed. It is found that the two wetting transition processes are irreversible for specific inherent contact angles and have different transition routes, the energy barrier exists on an ideally patterned surface and the gravity can be crucial to overcome the energy barrier and trigger the transition.
\end{abstract}

Keywords: wetting transitions, biomimetic surfaces, energy barrier, gravity effect, numerical study, lattice Boltzmann method

\section{Introduction}

Inspired from natural surfaces with the property of water repellence, such as lotus leaves, rose petals and so forth, the wetting phenomena on natural or manmade rough surfaces have drawn much of researchers' attentions over the past few decades [1-7]. After a considerable amount of work on theoretical and experimental investigations, it is already well understood that the roughness of surfaces, typically on the order of microns, plays a significant role in the enhancement of hydrophobicity [8-11]. The understanding of the wetting phenomena can be invaluable and the manufactured biomimetic surfaces with micro-structured roughness have been widely applied in industrial engineering including textile, coating, self-cleaning or 
surfaces involving surface-tension-induced motion [12-15]. A droplet with a lower contact angle on a flat surface can achieve contact angles up to approximately $170^{\circ}$ simply due to the geometrical patterning [16].

Droplets can exist mainly in two wetting states on an ideal square-post patterned surface, Wenzel state and Cassie-Baxter state [5]. The Wenzel state is the homogeneous wetting state, where the grooves caused by the surface roughness are penetrated with water, while the CassieBaxter state is the heterogeneous state where there is gas trapped between the droplet and the solid surface. Most of the theories describing wetting on rough surfaces over the past years are based on Young theory, Wenzel theory and Cassie-Baxter theory [17-19]. The Young equation reveals the relationship between surface tensions and contact angle on an ideal flat surface; for rough surfaces, Wenzel equation is used to calculate the apparent contact angle for the homogeneous wetting state; while Cassie-Baxter equation were proposed for the heterogeneous wetting state. Although the three theories can be used to explain wetting phenomena to some extent, it is not sufficient to use them to completely explain the mechanisms of wetting phenomena, such as the mechanism of wetting transitions between Wenzel and Cassie-Baxter wetting states, which is crucial for manufacturing superhydrophobic surfaces but still not fully understood [20]. Patankar, N. A. [21] studied the wetting transition from heterogeneous state to homogeneous state from the energy balance point of view, and discussed the energy barrier as well as the role of gravity in wetting transition via comparing his theory to Yoshimitsu et al.'s [22] experimental data. Gao N. et al. [23] analysed the equilibrium energy for both of the wetting states through a mathematical model. $\mathrm{Zu}$ Y. et al. [12,24] presented a modified roughness parameter and the critical transition angle. Gong W. et al. [25] discussed the transition energy curves theoretically based on the intermediate transition processes. The reverse transition, Wenzel-to-Cassie wetting transition were achieved experimentally by heating the rough surfaces to evaporate the droplet in the vicinity of the three phase line [26,27].

Having been developed for about three decades, the lattice Boltzmann method becomes a very import tool for modelling of single and multiphase flow in mesoscale [28]. Dupuis and Yeomans [29] firstly used the lattice Boltzmann method to study the droplet motions on superhydrophobic surfaces, and then followed by other researchers who studied the Cassie-toWenzel transition [30], contact angle hysteresis [31], droplet morphology [32], droplet influence[33] and droplet motion on hydrophobic surfaces [34,35]. A significant issue in most of their models is that the unwanted spurious currents cannot be eliminated and a large density ratio for liquid/gas system cannot be achieved. Connington et al. [36] proposed a new implementation of boundary conditions for the complex geometry in simulations of droplets on superhydrophobic surfaces, which can eliminate spurious currents, however, they did not accurately capture the transition between wetting states. Among all the lattice Boltzmann models, the phase field lattice Boltzmann model has been successfully applied in two phase flow simulation with large density ratio [37]. Yan \& $\mathrm{Zu}$ [38] presented a phase field lattice Boltzmann scheme for two-phase flows involving partial wetting surfaces and large density ratio up to 1000 which combines the advantages of the two models presented by Briant $[39,40]$ and Inamuro[41]. This model has been successfully used in simulation of the droplet motion on micro-structured rough surfaces [42,43]. Tanaka et al. [44] used this phase field model to conduct a 2-D simulation of dynamic behaviour of droplet on flat solid surfaces. 
The aim of this paper is using the phase field lattice Boltzmann method for large density ratio to study the wetting transitions between Wenzel state and Cassie-Baxter state on a square-post textured surface in mesoscale and to help understand the mechanism of wetting transitions.

\section{Numerical approach}

In this paper, the simulation is based on the phase field lattice Boltzmann approach for incompressible two-phase flows with large density ratio involving partial wetting surface [42]. As shown in Figure 1, the model is in three dimension and based on D3Q15 scheme. The velocity vectors are:

$$
\begin{aligned}
& {\left[\mathbf{c}_{0}, \mathbf{c}_{1}, \mathbf{c}_{2}, \mathbf{c}_{3}, \mathbf{c}_{4}, \mathbf{c}_{5}, \mathbf{c}_{6}, \mathbf{c}_{7}, \mathbf{c}_{8}, \mathbf{c}_{9}, \mathbf{c}_{10}, \mathbf{c}_{11}, \mathbf{c}_{12}, \mathbf{c}_{13}, \mathbf{c}_{14}\right]} \\
& =\left[\begin{array}{lllllllllllllllllll}
0 & 1 & 0 & 0 & - & 1 & 0 & 0 & 1 & -1 & 1 & 1 & -1 & 1 & -1 & -1 \\
0 & 0 & 1 & 0 & 0 & -1 & 0 & 1 & 1 & -1 & 1 & -1 & -1 & 1 & -1 \\
0 & 0 & 0 & 1 & 0 & 0 & -1 & 1 & 1 & 1 & -1 & -1 & -1 & -1 & 1
\end{array}\right]
\end{aligned}
$$

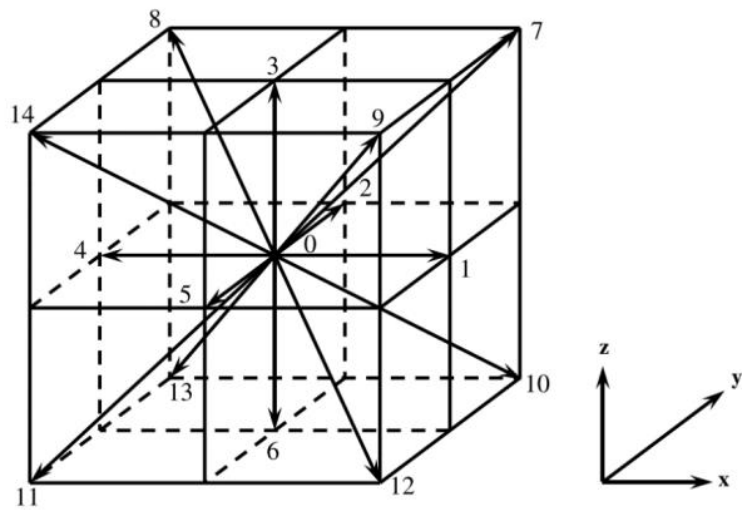

Fig. 1 D3Q15 scheme

Two velocity distribution functions, $f_{\alpha}$ and $g_{\alpha}$, are applied in this phase field model. The distribution function $f_{\alpha}$ is applied for the calculation of the order parameter $\phi$ which is used to distinguish the gas/liquid two phases. While distribution function $g_{\alpha}$ is employed as the distribution function of the predicted velocity $\boldsymbol{u}^{\prime}$, which needs to be corrected by pressure gradient of the two-phase system. The lattice Boltzmann equations including streaming and collision steps of particles can be written as:

$$
f_{\alpha}\left(\boldsymbol{x}+\mathbf{c}_{\alpha} \delta_{t}, t+\delta_{t}\right)=f_{\alpha}(\boldsymbol{x}, t)-\frac{1}{\tau_{f}}\left(f_{\alpha}(\boldsymbol{x}, t)-f_{\alpha}^{e q}(\boldsymbol{x}, t)\right)
$$

$$
\begin{aligned}
g_{\alpha}\left(\boldsymbol{x}+\mathbf{c}_{\boldsymbol{\alpha}} \delta_{t}, t\right. & \left.+\delta_{t}\right) \\
& =g_{\alpha}(\boldsymbol{x}, t)-\frac{1}{\tau_{g}}\left(g_{\alpha}(\boldsymbol{x}, t)-g_{\alpha}^{e q}(\boldsymbol{x}, t)\right)+3 \omega_{\alpha} \frac{1}{\rho} \nabla \cdot[\mu(\nabla \boldsymbol{u}+\boldsymbol{u} \nabla)] \\
& \cdot \mathbf{c}_{\boldsymbol{\alpha}}
\end{aligned}
$$

Where $\boldsymbol{x}$ and $t$ are space position and time; $\boldsymbol{u}, \mu$, and $\rho$ are the macroscopic parameters, representing velocity, dynamic viscosity and density; $\tau_{f}$ and $\tau_{g}$ are dimensionless single relaxation times; $\delta_{t}=1$ is the time step; $f_{\alpha}^{e q}$ and $g_{\alpha}^{e q}$ are the equilibrium distribution functions for equilibrium states and defined as: 


$$
\begin{array}{r}
f_{\alpha}^{e q}(\boldsymbol{x}, t)=\mathrm{H}_{\alpha} \phi+F_{\alpha}\left[p_{0}-\mathrm{k}_{\mathrm{f}} \phi \nabla^{2} \phi-\frac{\mathrm{k}_{\mathrm{f}}}{6}|\nabla \phi|^{2}\right]+3 \omega_{\alpha} \phi\left(\mathbf{c}_{\alpha}^{\prime} \cdot \boldsymbol{u}\right)+\omega_{\alpha} \mathrm{k}_{\mathrm{f}} \mathbf{c}_{\alpha}^{\prime} \cdot \boldsymbol{G}(\phi) \\
\cdot \mathbf{c}_{\boldsymbol{\alpha}} \\
g_{\alpha}^{e q}(\boldsymbol{x}, t)=\omega_{\alpha}\left[1+3\left(\mathbf{c}_{\alpha}^{\prime} \cdot \boldsymbol{u}\right)+\frac{9}{2}\left(\mathbf{c}_{\alpha}^{\prime} \cdot \boldsymbol{u}\right)^{2}-\frac{3}{2} \boldsymbol{u}^{2}+\frac{3}{2}\left(\tau_{g}-\frac{1}{2}\right) \mathbf{c}_{\alpha}^{\prime} \cdot(\nabla \boldsymbol{u}+\boldsymbol{u} \nabla) \cdot \mathbf{c}_{\alpha}\right] \\
+\omega_{\alpha} \frac{k_{f}}{\rho} \mathbf{c}_{\alpha}^{\prime} \cdot \boldsymbol{G}(\phi) \cdot \mathbf{c}_{\alpha}-\frac{2}{3} \mathrm{~F}_{\alpha} \frac{\mathrm{k}_{\mathrm{f}}}{\rho}|\nabla \phi|^{2}(5)
\end{array}
$$

where

$$
\begin{gathered}
\omega_{\alpha}=\left\{\begin{aligned}
\frac{2}{9} & \alpha=0 \\
\frac{1}{9} & \alpha=1, \ldots 6 \\
\frac{1}{72} & \alpha=7, \ldots, 14
\end{aligned}\right. \\
\mathrm{F}_{\alpha}=\left\{\begin{aligned}
-\frac{7}{3} & \alpha=0 \\
\frac{1}{3} & \alpha=1, \ldots 6 \\
\frac{1}{24} & \alpha=7, \ldots, 14
\end{aligned}\right. \\
\mathrm{H}_{\alpha}=\left\{\begin{array}{ll}
1, & \alpha=0 \\
0, & \alpha
\end{array}=1, \ldots, 14\right.
\end{gathered}
$$

and

$$
\boldsymbol{G}(\phi)=\frac{9}{2}(\nabla \phi)(\phi \nabla)-\frac{3}{2}|\nabla \phi|^{2} \mathbf{I}
$$

and as a constant, $\mathrm{k}_{\mathrm{f}}$ is related to the strength of surface tension as well as the width of interface. With $\Psi(\phi)$ as the bulk free-energy density, the equation of state of the fluid is:

$$
p_{0}=\phi \frac{\partial \Psi}{\partial \phi}-\Psi
$$

The macroscopic variables $\phi, \boldsymbol{u}^{\prime}, \rho$ and $\mu$ can be computed from the distribution functions by:

$$
\begin{aligned}
& \phi=\sum_{\alpha} f_{\alpha}, \boldsymbol{u}^{\prime}=\sum_{\alpha} \mathbf{c}_{\alpha} g_{\alpha} \\
& \rho=\left\{\begin{aligned}
\rho_{G}, & \phi<\phi_{G} \\
\frac{\phi-\phi_{G}}{\phi_{L}-\phi_{G}}\left(\rho_{L}-\rho_{G}\right)+\rho_{G}, & \phi_{G} \leq \phi \leq \phi_{L} \\
\rho_{L}, & \phi>\phi_{L}
\end{aligned}\right. \\
& \mu=\frac{\rho-\rho_{G}}{\rho_{L}-\rho_{G}}\left(\mu_{L}-\mu_{G}\right)+\mu_{G}
\end{aligned}
$$


where the subscript $\mathrm{L}$ means liquid and $\mathrm{G}$ represents gas.

In this model, the precalculated velocity $\boldsymbol{u}^{\prime}$ does not meet the continuity condition $(\nabla \cdot \boldsymbol{u}=\mathbf{0})$, so $\boldsymbol{u}^{\prime}$ should be modified for a divergence free velocity by:

$$
\begin{gathered}
\boldsymbol{u}-\boldsymbol{u}^{\prime}=-\frac{\nabla p}{\rho} \\
\nabla \cdot \boldsymbol{u}^{\prime}=\nabla \cdot\left(\frac{\nabla p}{\rho}\right)
\end{gathered}
$$

where $p$ is the pressure. The Poisson equation is solved in this paper in the framework of lattice Boltzmann method:

$$
h_{\alpha}\left(\boldsymbol{x}+\mathbf{c}_{\boldsymbol{\alpha}}, m+\mathbf{1}\right)=h_{\alpha}(\boldsymbol{x}, m)-\frac{1}{\tau_{h}}\left[h_{\alpha}(\boldsymbol{x}, m)-\omega_{\alpha} p(\boldsymbol{x}, m)\right]-\frac{\omega_{\alpha}}{3} \nabla \cdot \boldsymbol{u}^{\prime}
$$

where $\tau_{h}=0.5+1 / \rho$, and $m$ is the iteration number. The iterated pressure can be calculated as:

$$
p(\boldsymbol{x}, m+1)=\sum_{\alpha} h_{\alpha}(\boldsymbol{x}, m+1)
$$

and the iteration residual should meet the following condition before the modified velocity can be used for calculation:

$$
|p(\boldsymbol{x}, m+1)-p(\boldsymbol{x}, m)|<\varepsilon
$$

Landau free-energy equation is used in this model for partial wetting boundary condition:

$$
\Psi=\int_{V} d V\left[\Psi(\phi)+\frac{\mathrm{k}_{\mathrm{f}}(\nabla \phi)^{2}}{2}\right]
$$

The free-energy density $\Psi(\phi)$ can be rewritten as follows in terms of isothermal system:

$$
\Psi(\phi)=\beta\left(\phi-\phi_{G}\right)^{2}\left(\phi-\phi_{L}\right)^{2}+\mu_{b} \phi-p_{b}
$$

where $\beta$ is a constant parameter concerned with the interfacial thickness; $p_{b}$ is the bulk pressure. The chemical potential is defined as

$$
\mu_{c}=\frac{\partial \Psi}{\partial \phi}=4 \beta\left(\phi-\phi_{L}\right)\left(\phi-\phi_{G}\right)\left(\phi-\phi_{M}\right)+\mu_{b}
$$

where $\phi_{M}=\left(\phi_{L}+\phi_{G}\right) / 2 ; \mu_{b}$ is the bulk chemical potential.

By combination of Eq. (19) and Eq. (8), here is

$$
p_{0}=\beta\left(\phi-\phi_{L}\right)\left(\phi-\phi_{G}\right)\left(3 \phi^{2}-\phi \phi_{L}-\phi \phi_{G}-\phi_{L} \phi_{G}\right)+p_{b}
$$

The interface thickness $D$ is represented as

$$
D=\frac{4}{\phi_{L}-\phi_{G}} \sqrt{\frac{\mathrm{k}_{\mathrm{f}}}{2 \beta}}
$$

The liquid-gas surface tension $\sigma_{L G}$ is given by 


$$
\sigma_{L G}=\frac{\left(\phi_{L}-\phi_{G}\right)^{3}}{6} \sqrt{2 \mathrm{k}_{\mathrm{f}} \beta}
$$

Given that wetting potential

$$
\Omega=\frac{4 \lambda}{\left(\phi_{L}-\phi_{G}\right)^{2} \sqrt{2 \mathrm{k}_{\mathrm{f}} \beta}}
$$

The following equations for surface tensions can be obtained, and details for the derivation of surface tensions can be found from $\mathrm{Zu}$ Y.Q. [42]

$$
\begin{gathered}
\sigma_{S G}=-\lambda \frac{\phi_{L}+\phi_{G}}{2}+\frac{\sigma_{L G}}{2}-\frac{\sigma_{L G}}{2}(1-\Omega)^{\frac{3}{2}} \\
\sigma_{S L}=-\lambda \frac{\phi_{L}+\phi_{G}}{2}+\frac{\sigma_{L G}}{2}-\frac{\sigma_{L G}}{2}(1+\Omega)^{\frac{3}{2}}
\end{gathered}
$$

The wetting angle then can be given as

$$
\cos \theta_{Y}=\frac{\left[(1+\Omega)^{\frac{3}{2}}-(1-\Omega)^{\frac{3}{2}}\right]}{2}
$$

For a given wetting angle the wetting potential $\Omega$ can be calculated as

$$
\Omega=2 \operatorname{sgn}\left(\frac{\pi}{2}-\theta_{Y}\right)\left\{\cos \left(\frac{\gamma}{3}\right)\left[1-\cos \left(\frac{\gamma}{3}\right)\right]\right\}^{\frac{1}{2}}
$$

Where

$$
\gamma=\arccos \left(\sin ^{2} \theta_{Y}\right)
$$

and $\operatorname{sgn}(\xi)$ is to give the sign of $\xi$.

To implement the partial wetting boundary condition for the phase field lattice Boltzmann model, the order parameter gradient near the wall should satisfy the follow condition:

$$
\boldsymbol{n} \cdot \nabla \phi=-\frac{\lambda}{\mathrm{k}_{\mathrm{f}}}
$$

where $\boldsymbol{n}$ is the unit vector denoting the normal direction of the wall.

\section{Results and discussion}

The droplet motion on a square-post patterned micro surface, as shown in Fig. 2, is simulated in a liquid/gas two-phase system. The roughness parameters in this model are set as $\mathrm{a}=\mathrm{b}=$ $\mathrm{h}=5 \mu \mathrm{m} .-3 \omega_{\alpha} \mathbf{c}_{\alpha 3}\left(1-\rho_{G} / \rho\right) \mathrm{g}$ is added to the right side of Eq. (5) for simulation of gravity effect, and $g$ means the nondimensional gravitational acceleration. The densities of liquid and gas are $\rho_{L}=1000, \rho_{G}=1.29$, and the viscosities of the two phase fluids are $\mu_{L}=10, \mu_{G}=$ 0.1935 , respectively. To achieve the real liquid/gas two phase system, the physical parameters are calculated with the transformational relations between the lattice units and the physical units: the lattice length unit $L_{0}=1 \times 10^{-6} \mathrm{~m}$, the lattice time unit $T_{0}=1 \times 10^{-8} \mathrm{~s}$, and the lattice mass unit $M_{0}=1 \times 10^{-18} \mathrm{~kg}$. With the transformational relations, the natural dimensional physical parameters can be obtained: $\overline{\rho_{L}}=1000 \mathrm{~kg} \mathrm{~m}^{-3}, \overline{\rho_{G}}=1.29 \mathrm{~kg} \mathrm{~m}^{-3}, \overline{\mu_{L}}=$ 
$1 \times 10^{-3} \mathrm{~kg} \mathrm{~m}^{-1} \mathrm{~s}^{-1}, \overline{\mu_{G}}=1.935 \times 10^{-5} \mathrm{~kg} \mathrm{~m}^{-1} \mathrm{~s}^{-1}$ and $\overline{\mathrm{g}}=9.8 \mathrm{~m} \mathrm{~s}^{-2}$. The initial diameter of a spherical droplet is $60 \mu \mathrm{m}$. The computational domain including solid surface and liquid droplet surrounded by gas are divided into $37 \times 37 \times 75$, which is quarter of the whole simulation region with symmetrical boundary condition applied. Unless otherwise specified, within a cuboid computational domain, the boundary conditions for the following simulation are set as: free outflow/inflow boundary conditions on the left, front and upper surfaces, symmetrical boundary conditions on the right and back surfaces, and non-slip boundary condition on the solid surfaces. The residual in Eq. (16) is $\varepsilon=1 \times 10^{-6}$. The apparent contact angles for a droplet can be calculated by the following equations:

Young equation:

$$
\cos \theta_{Y}=\frac{\sigma_{S G}-\sigma_{S L}}{\sigma_{L G}}
$$

Wenzel equation:

$$
\cos \theta_{w}=r \cos \theta_{Y}
$$

Cassie-Baxter equation:

$$
\cos \theta_{C B}=r_{f} f \cos \theta_{Y}+f-1
$$

Critical angle:

$$
\cos \theta_{C}=\frac{1-f}{r_{f} f-r}
$$

where $\sigma$ is the surface tension which represents the energy per unit area of the interface between solid/gas, solid/liquid or liquid/gas, $f$ is the area fraction on the horizontal projected plane of the liquid-solid contact over the total area of solid-liquid and liquid-gas contact, $r_{f}$ is the ratio of the actual wetted area over the projected area, and $r$ is the ratio of the actual surface area with respect to the projected structure surface. The critical Young's angle calculated by Eq. (43) in our model is $115.4^{\circ}$, therefore Young's angles of $105^{\circ}$ for Wenzel state preferable and $130^{\circ}$ for Cassie-Baxter state preferable are tested in this paper. The following figures in 2$\mathrm{D}$ view are the density distributions on the cross section where y-coordinate is $36 \mu \mathrm{m}$.

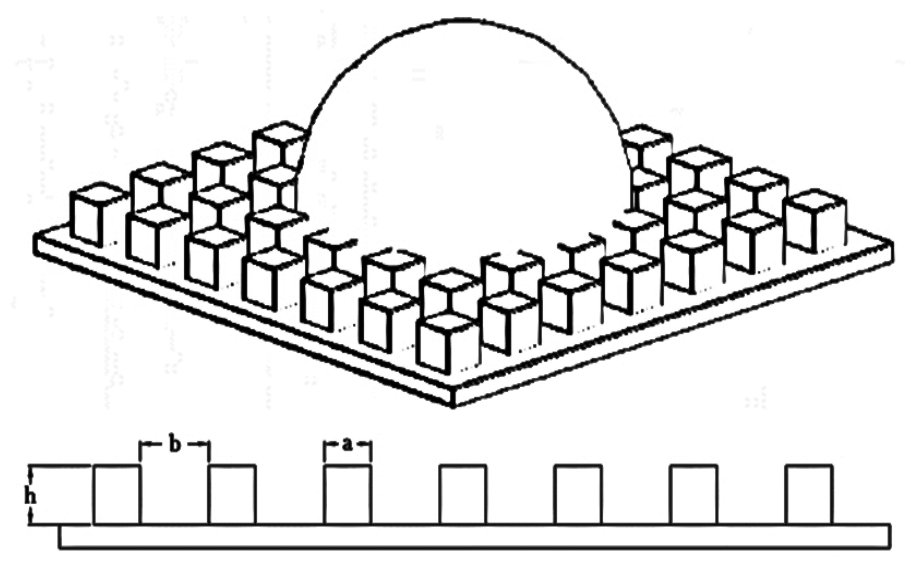

Fig. 2 Structure of the square-post patterned micro surface 


\subsection{Validation of the numerical model}

To validate the numerical model, firstly the apparent contact angles of water droplets on a flat surface are tested after the two phase systems reach static equilibrium in a $37 \times 37 \times 56$ computational domain. As shown in Fig. 3, the equilibrium contact angles are approximately $105^{\circ}$ and $130^{\circ}$, which are almost the same as previously set. Following the apparent contact angles of water droplets on a square-post patterned surface are tested as well, as shown in Fig. 4. For the first case where Young's angle is $105^{\circ}$, smaller than the critical angle, the wetting state is Wenzel state with the apparent contact angle of $119^{\circ}$ in Fig. 4(a), and the theoretical contact angle calculated by Eq. (41) is $121.2^{\circ}$; while when Young's angle is $130^{\circ}$ and the wetting state is Cassie-Baxter state preferable, the simulated equilibrium apparent contact angle in Fig. 4(b) is $160^{\circ}$, which is also very close to the theoretical value of $155.6^{\circ}$ by Eq. (42). Fig. 4(c) and Fig. 4(d) present the droplets in 3-D view, from where it can be clearly seen that the grooves caused by roughness is waterlogged for the Wenzel state, while there is gas trapped between water liquid and solid surface for the Cassie-Baxter state. Hence, the numerical model can be used to study the wetting phenomena.

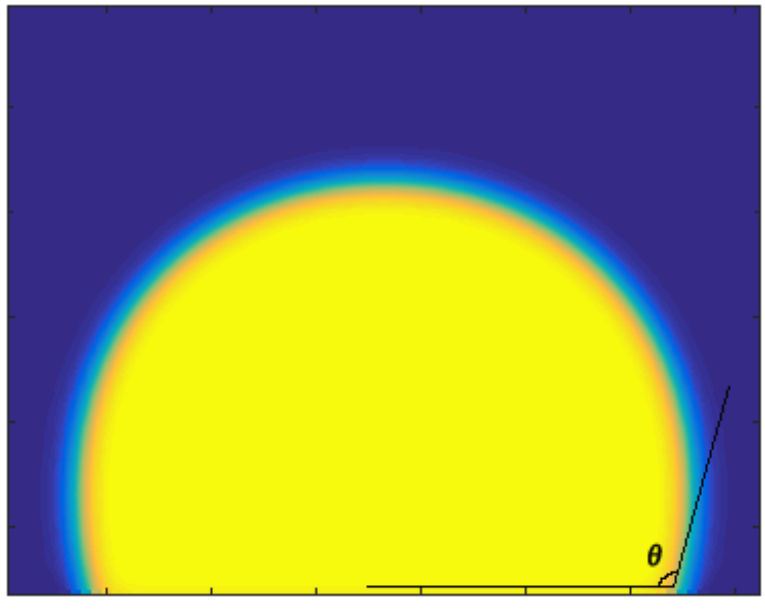

(a)

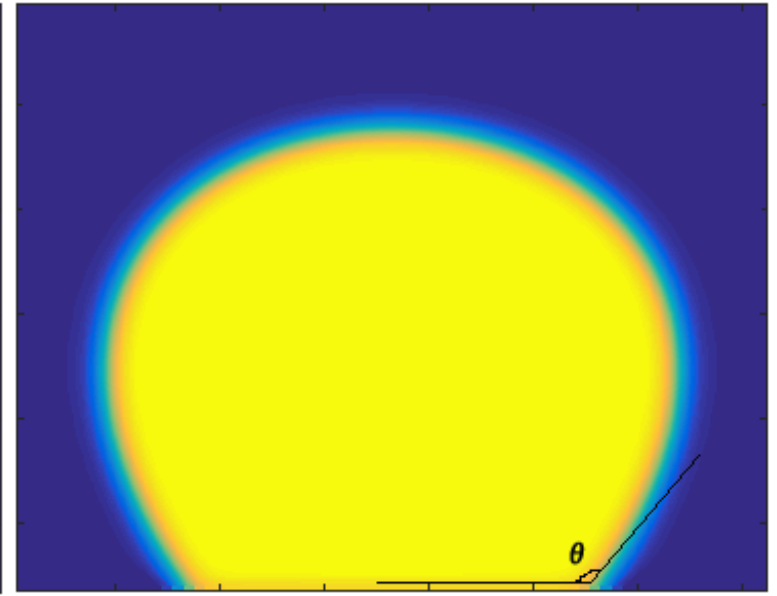

(b)

Fig. 3 Droplets on flat surfaces with different Young's angles (a) $\theta_{Y}=105^{\circ}$ (b) $\theta_{Y}=130^{\circ}$ 


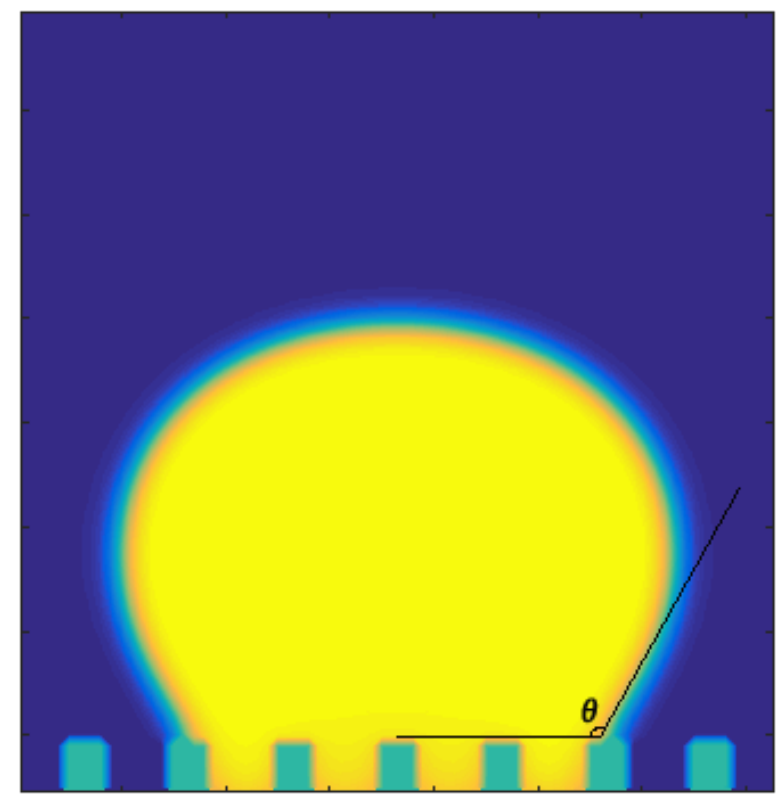

(a)

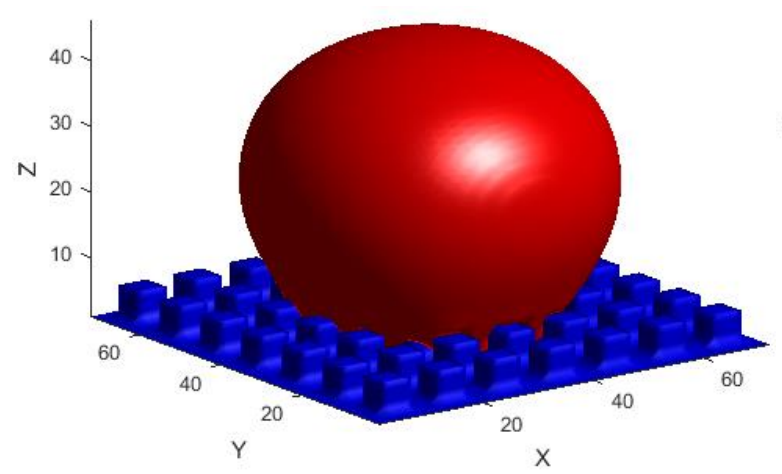

(c)

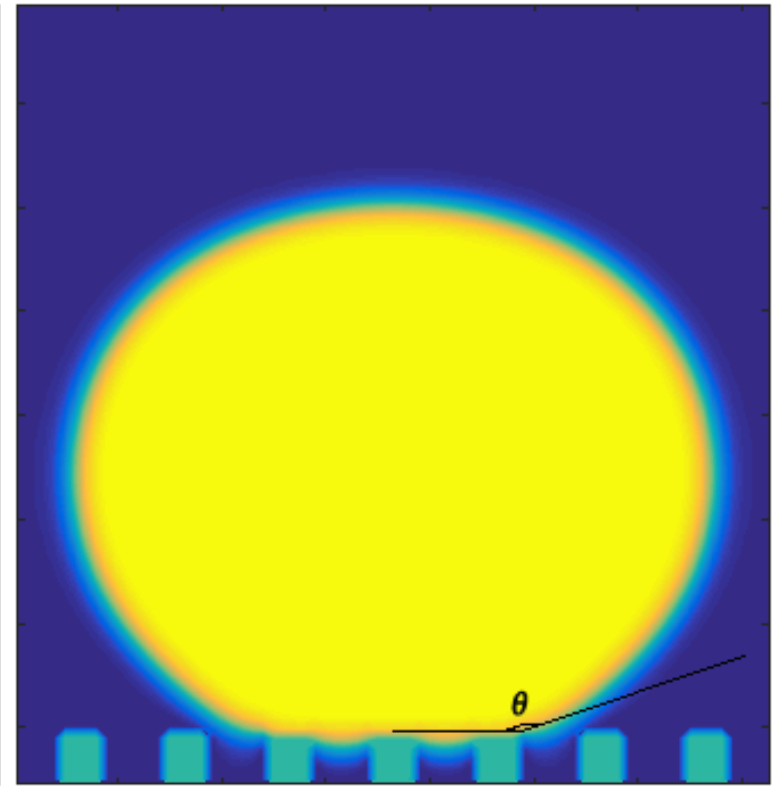

(b)

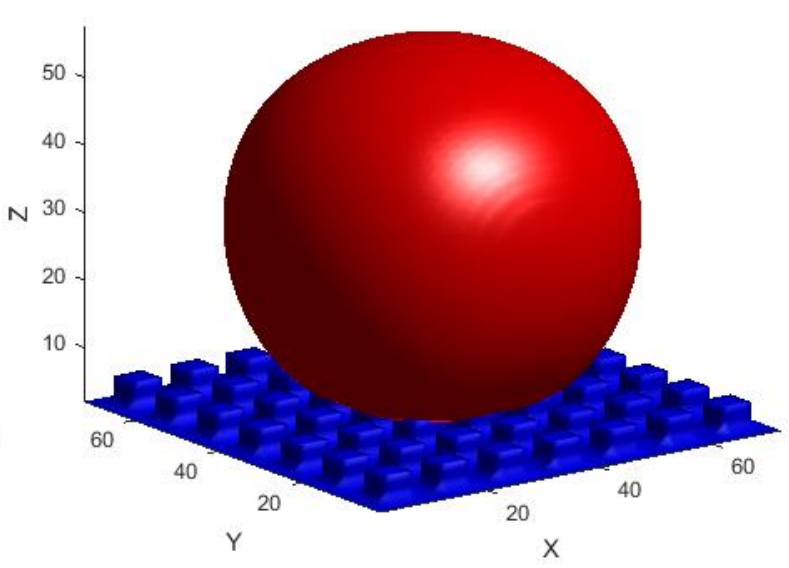

(d)

Fig. 4 Droplets on square-post patterned surfaces with different Young's angles (a) $\theta_{Y}=$ $105^{\circ}, 2-\mathrm{D}$ view (b) $\theta_{Y}=130^{\circ}, 2-\mathrm{D}$ view (c) $\theta_{Y}=105^{\circ}, 3-\mathrm{D}$ view (d) $\theta_{Y}=130^{\circ}, 3-\mathrm{D}$ view

\subsection{Wetting transitions}

The Cassie-to-Wenzel wetting state transition is firstly simulated with a Wenzel state preferable Young's angle of $105^{\circ}$, as shown in Fig. 5. To overcome the energy barrier[5], an initial velocity of $0.001 \mathrm{~m} / \mathrm{s}$ is given and the initial height of the centre of the droplet is $60 \mu \mathrm{m}$. From the pictures it can be seen that after about $1 \mathrm{~ms}$ of the initial condition the droplet touches the upper surface of the square posts, presenting a Cassie-Baxter wetting state, and then the transition occurs. While the transition is in process, the droplet lower surface moves downward, keeping nearly horizontal until the inner parts touch the bottom solid surface. After approximately $2.4 \mathrm{~ms}$ the transition process is completed. In the $1.4 \mathrm{~ms}$ of the wetting transition process from Cassie-Baxter state to Wenzel state $(1.00 \mathrm{~ms}-2.40 \mathrm{~ms})$, the time spent in moving down is about $1.34 \mathrm{~ms}$ while the remaining $0.06 \mathrm{~ms}$ is for soaking the bottom solid surface, which means the latter process diminishes the free energy much more, in another word, the energy barrier is main exist in the moving down process. This is consistent with the theoretical 
analysis in Patankar, N. A. [45] Moreover, in our simulation the Cassie-to-Wenzel transition occurs only by depinning of the three-phase line, but the "sag transition" with pinning the threephase line, which was predicted by Patankar, N. A. [46] was not observed.

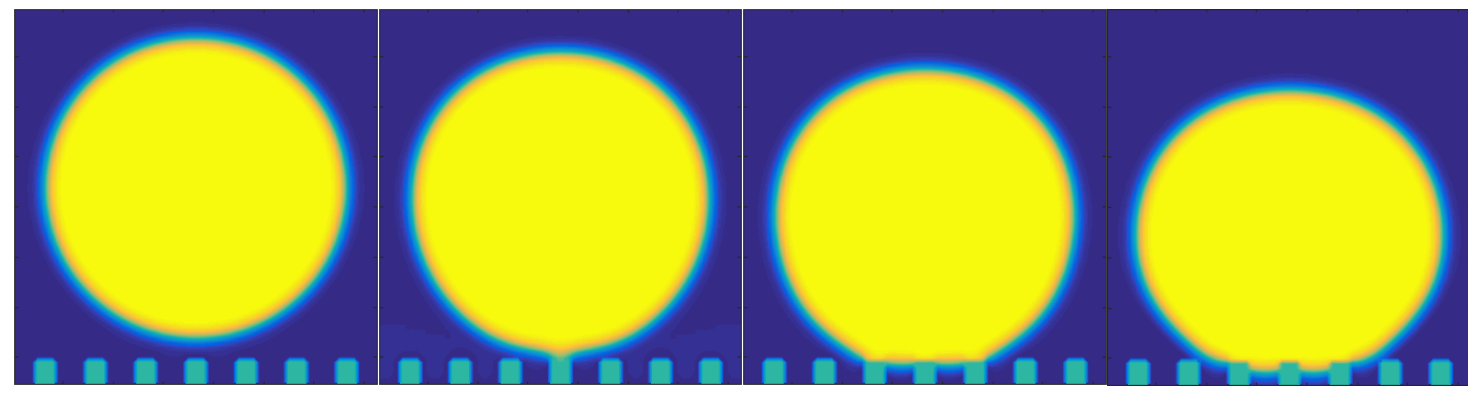

$$
t=0.00 \mathrm{~ms} \quad t=0.60 \mathrm{~ms} \quad t=1.00 \mathrm{~ms} \quad t=1.50 \mathrm{~ms}
$$

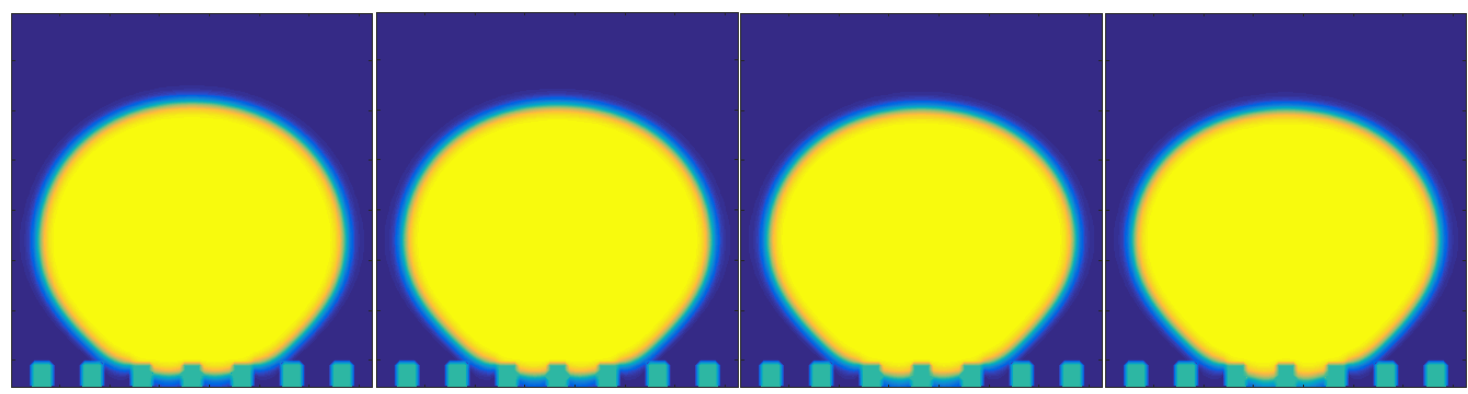

$$
t=1.80 \mathrm{~ms} \quad t=2.00 \mathrm{~ms} \quad t=2.20 \mathrm{~ms} \quad t=2.30 \mathrm{~ms}
$$

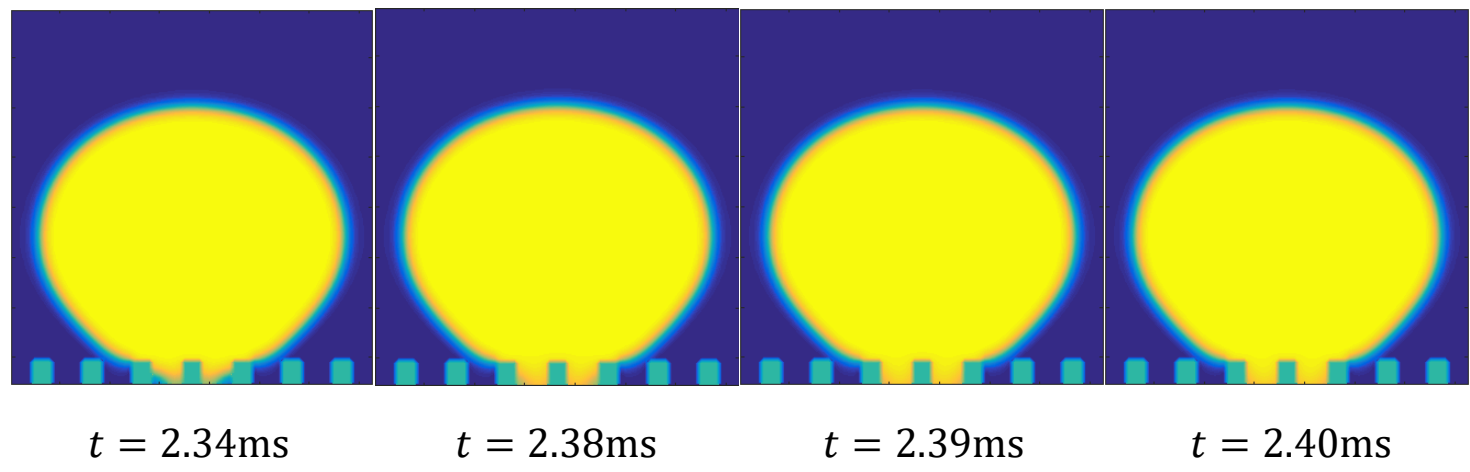

Fig. 5 Cassie-to-Wenzel wetting transition process, $\theta_{Y}=105^{\circ}$

The reverse transition from Wenzel state to Cassie-Baxter state, which is difficult to implement by experiment due to the unachievable initial state, is then tested with a Cassie-Baxter state preferable Young's angle of $130^{\circ}$ in the numerical simulation, as shown in Fig. 6. The droplet is placed on the patterned surface with an initial condition of Wenzel state with water filled in the grooves, as shown in the first picture when $t=0 \mathrm{~ms}$. It can be seen that the reverse transition route is quite different from the Cassie-to-Wenzel transition, which has a moving down process followed by the soaking bottom surface process. The reverse transition proceeds from the outer side of the post grooves to the inner side, and the liquid-solid-gas triple line moves in both horizontal and vertical directions. The whole transition takes places in around $1.60 \mathrm{~ms}$. This result indicate that the Wenzel-to-Cassie wetting transition process can occur 
spontaneously without any external forces such as heating so long as the inherent contact angle is large enough.

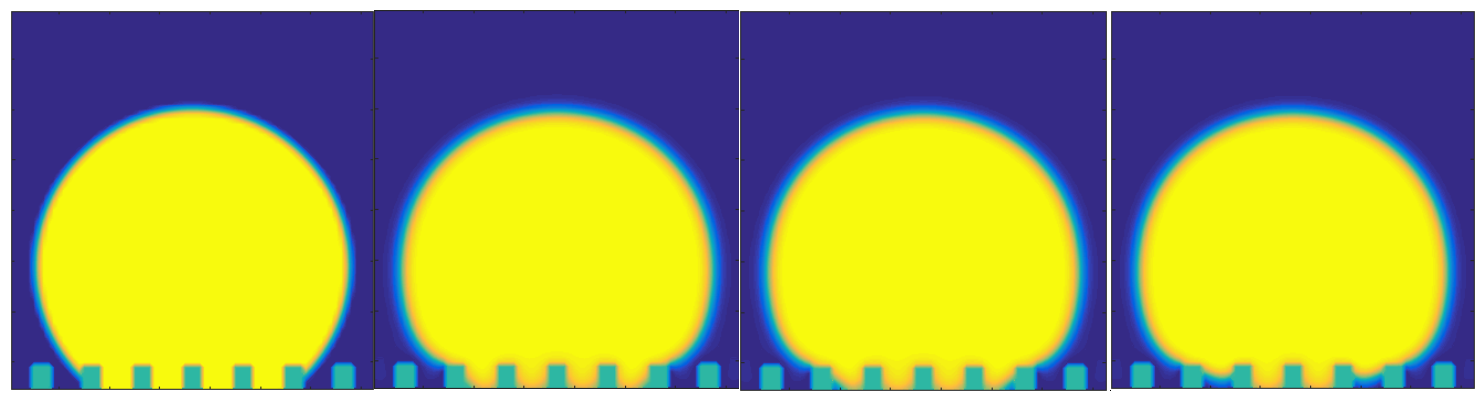

$$
t=0.00 \mathrm{~ms} \quad t=0.50 \mathrm{~ms} \quad t=0.60 \mathrm{~ms} \quad t=0.70 \mathrm{~ms}
$$

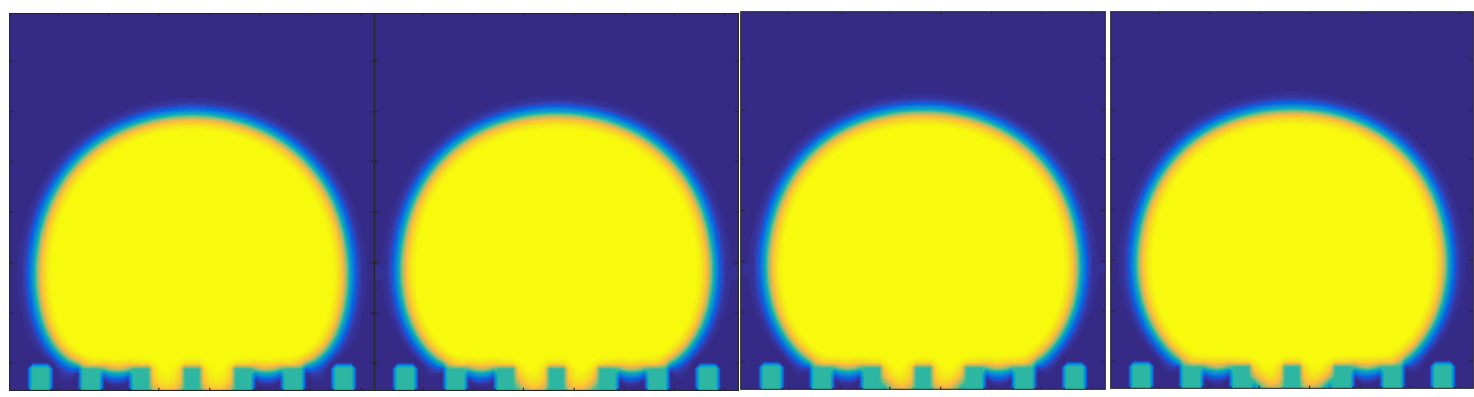

$$
t=0.80 \mathrm{~ms} \quad t=1.10 \mathrm{~ms} \quad t=1.20 \mathrm{~ms} \quad t=1.30 \mathrm{~ms}
$$

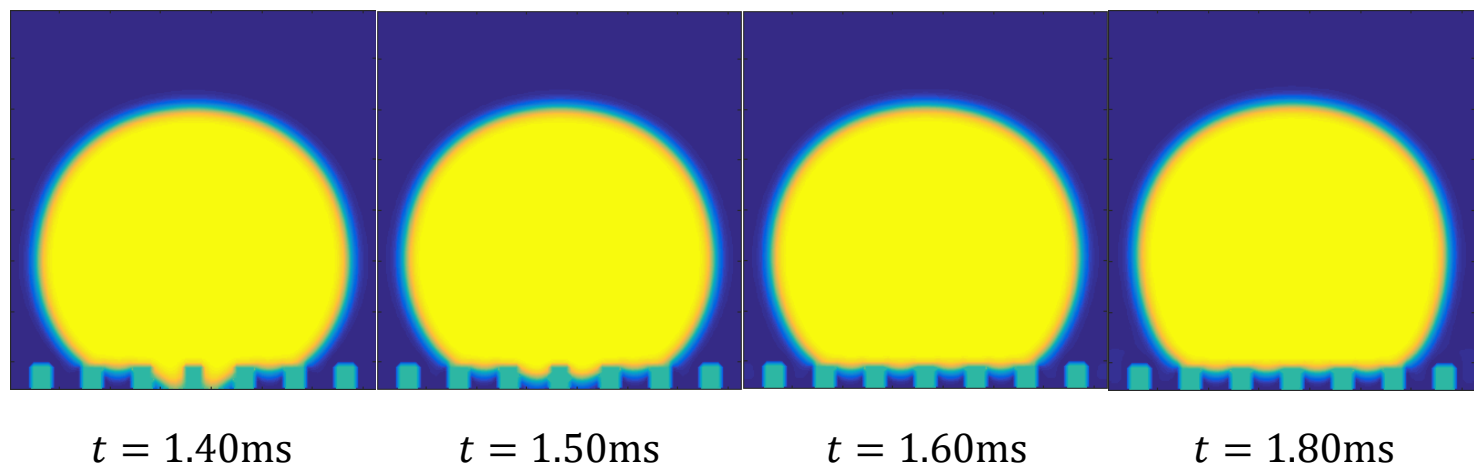

Fig. 6 Wenzel-to-Cassie wetting transition process, $\theta_{Y}=130^{\circ}$

It is generally agreed that the wetting transitions are irreversible [47]. For each case of our simulation work, the transition occurs along the same direction, from Cassie-Baxter state to Wenzel state for a large Young's angle or the reverse transition for a smaller Young's angle, irreversible when no extra forces are loaded to the droplets.

\subsection{Energy barrier}

Although it has been observed in experiments that the Wenzel state and Cassie-Baxter state of droplets can coexist on the same surface [48], the manufactured surface cannot be as ideal as people expect, and the roughness of the manufactured posts or the hierarchy structure that cannot be eliminated completely may have influence on the existence of the energy barrier. However, in the numerical simulation there is no such a problem, as all the posts are ideally patterned. Two droplets are placed on the same surface with the same Young's angle of $105^{\circ}$, 
with Wenzel wetting state and Cassie-Baxter wetting sate as the initial wetting conditions, respectively. No external forces such as initial velocity or pressure but gravity is loaded to the droplets. When achieving equilibrium states, as shown in Fig. 7, the Wenzel state and CassieBaxter state coexist, and no wetting transition happens spontaneously between the two droplets. This wetting phenomenon is firstly achieved by numerical simulation in our paper, and it proves that the energy barrier exists from the simulation point of view by eliminating the factor of roughness on each post of any manufactured surface.

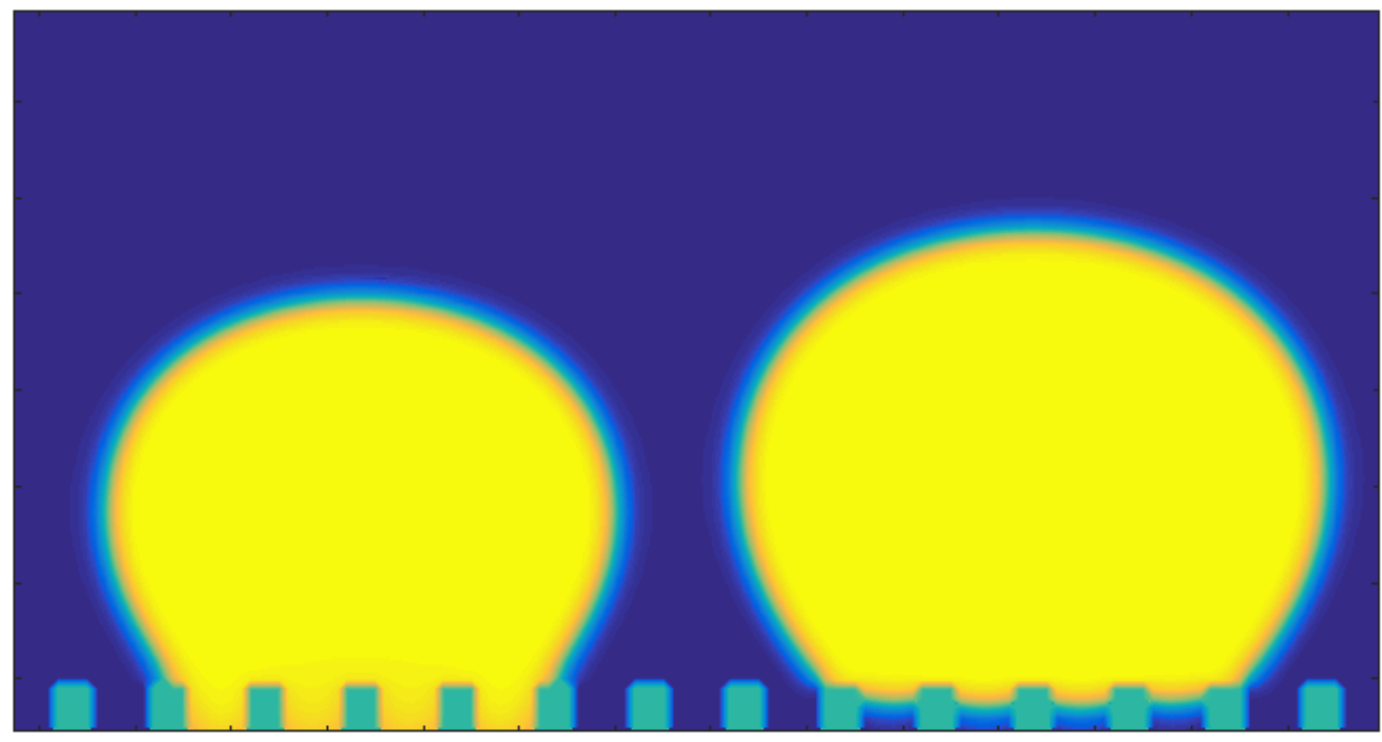

Fig. 7 Wenzel state and Cassie-Baxter state coexist on the same surface, $\theta_{Y}=105^{\circ}$

\subsection{Gravity effect on wetting transition}

In the microscale, gravity does not have an significant effect on the shape of a water droplet, but may be crucial to wetting transition.[45] During the Cassie-to-Wenzel transition process, the barycentre of the droplet declines, causing a decrease of the energy of the droplet, which can be a factor that overcomes the energy barrier. However, the gravity effect on wetting transition cannot be approved by experiments. In the numerical simulation, gravity can be loaded on the droplet or not just by changing the value of acceleration of gravity. Fig. 8 presents a specific case that the wetting transition is affected by the gravity effect when $\theta_{Y}=102^{\circ}$ after $0.90 \mathrm{~ms}$. Gravity is not loaded to the system in Fig. 8(a) and loaded in Fig. 8(b). It can be seen that after the same period of time, the wetting transition occurs for the droplet involving gravity but the other droplet is still in a Cassie-Baxter state. Although in our simulation the droplet without gravity in Fig. 8(a) finally transited to Wenzel state, which may be triggered by the pseudo currents on the wall or a small velocity field caused by initialization, the results still indicate that gravity has a significant effect on wetting transition because the transition occurs much faster for the droplet with gravity. 


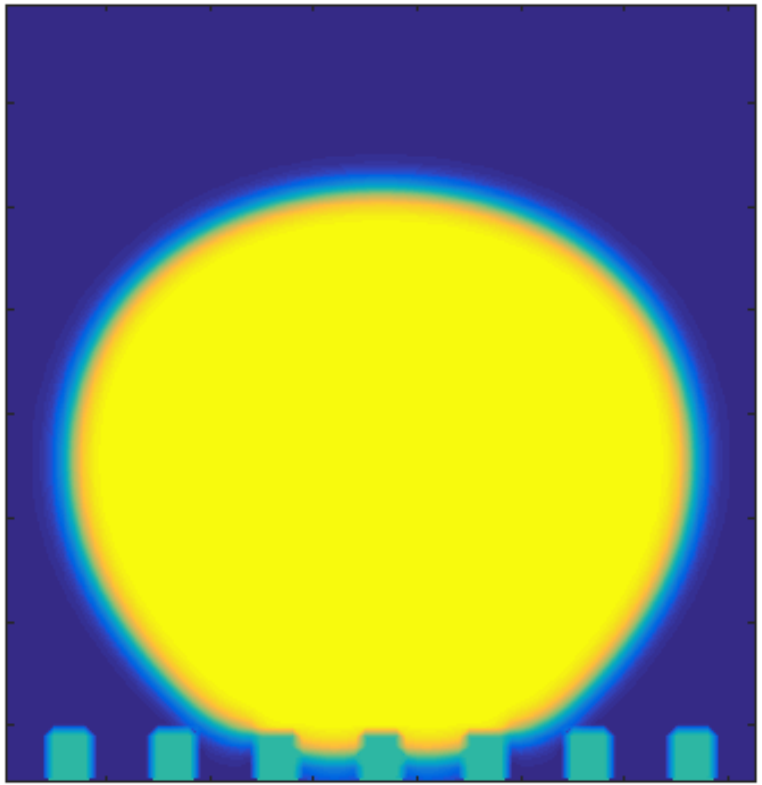

(a)

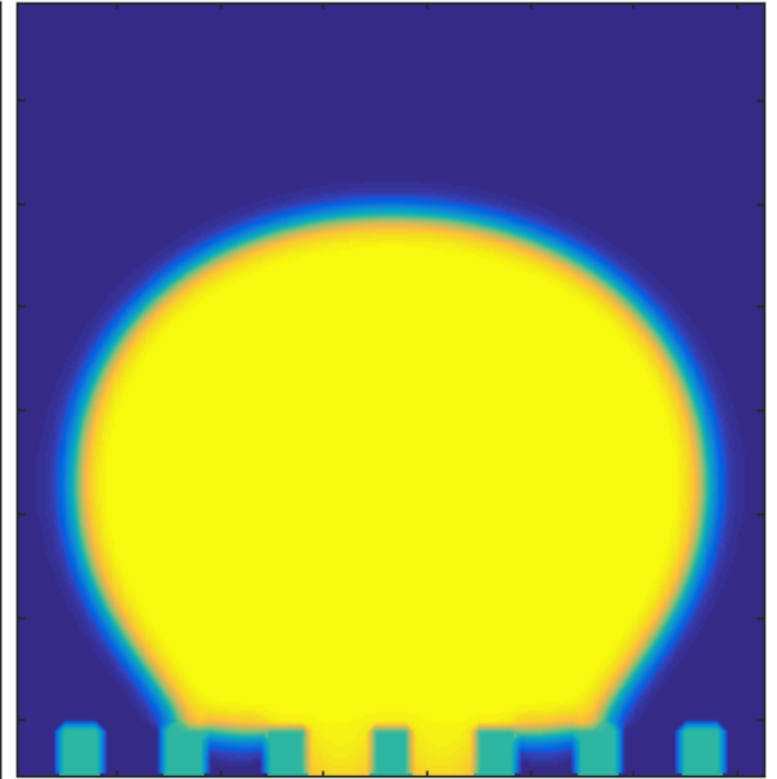

(b)

Fig. 8 Gravity effect on wetting transition, $\theta_{Y}=102^{\circ}, t=0.90 \mathrm{~ms}$ (a) without gravity (b) with gravity

\section{Conclusions}

In this paper, the water droplet wetting transitions on square-patterned surfaces by mimicking the natural hydrophobic surfaces, which are difficult to be observed in experimental investigations, are achieved with a phase field lattice Boltzmann method in a two-phase system with large density ratio. The current work is very helpful in understanding the mechanism of wetting transitions, which is crucial for the development of superhydrophobic surfaces. The numerical model is firstly validated by comparing the apparent contact angles of both homogeneous and heterogeneous wetting states with the famous Wenzel equation and CassieBaxter equation, and the simulation results are in good agreement with the theoretical results. Then the Cassie-to-Wenzel wetting transition and the Wenzel-to-Cassie transition processes, the energy barrier and the gravity effect on wetting transition are studied. Following conclusions can be drawn from the numerical investigation:

(1) For the Cassie-to-Wenzel transition, it takes much more time of the droplet on moving down along the posts side surfaces than soaking the bottom solid surface, which means the energy barrier is mainly on the vertical transition process.

(2) The Wenzel-to-Cassie transition can be achieved spontaneously if an initial condition of homogeneous wetting state can be given on a surface with large Young's angle.

(3) Cassie-to-Wenzel transition and the reverse transition have different routes: the former occurs mainly in the vertical direction, while the reverse transition is primarily an outer-toinner process.

(4) For the cases tested in our work, the wetting transitions are irreversible without any external forces. 
(5) The two wetting states can coexist on a Wenzel state preferable surface excluding the effect from smaller scale of roughness than the scale of posts for manufactured surfaces, which means the energy barrier exists.

(6) Although gravity does not have a significant influence on the shape of the droplet in microscale, it can be a crucial factor to wetting transition.

\section{Acknowledgement}

The authors would like to acknowledge the financial support of this work by the doctoral degree scholarship of China Scholarship Council (CSC) and the University of Nottingham, UK. The authors also thank Dr Yingqing Zu from Fudan University, China for his kind help for lattice Boltzmann method.

\section{References}

[1] Sefiane, K. On the formation of regular patterns from drying droplets and their potential use for bio-medical applications. Journal of Bionic Engineering, 2010, 7, S82-S93.

[2] Fang, Y.; Sun, G.; Cong, Q.; Chen, G.-h.; Ren, L.-q. Effects of methanol on wettability of the non-smooth surface on butterfly wing. Journal of Bionic Engineering, 2008, 5, 127-133.

[3] Lai, H.; Ma, C. The lattice Boltzmann model for the second-order Benjamin-Ono equations. Journal of Statistical Mechanics: Theory and Experiment, 2010, 2010, P04011.

[4] Latt, J.; Chopard, B. Lattice Boltzmann method with regularized pre-collision distribution functions. Mathematics and Computers in Simulation, 2006, 72, 165-168.

[5] Yan, Y. Y.; Gao, N.; Barthlott, W. Mimicking natural superhydrophobic surfaces and grasping the wetting process: a review on recent progress in preparing superhydrophobic surfaces. Advances in Colloid and Interface Science, 2011, 169, 80-105.

[6] Bormashenko, E. Wetting transitions on biomimetic surfaces. Philosophical Transactions of the Royal Society of London A: Mathematical, Physical and Engineering Sciences, 2010, 368, 4695-4711.

[7] Byun, D.; Hong, J.; Ko, J. H.; Lee, Y. J.; Park, H. C.; Byun, B.-K.; Lukes, J. R. Wetting characteristics of insect wing surfaces. Journal of Bionic Engineering, 2009, 6, 63-70.

[8] Wong, T.-S.; Huang, A. P.-H.; Ho, C.-M. Wetting behaviors of individual nanostructures. Langmuir, 2009, 25, 6599-6603.

[9] Grunau, D.; Chen, S.; Eggert, K. A lattice Boltzmann model for multiphase fluid flows. Physics of Fluids A: Fluid Dynamics (1989-1993), 1993, 5, 2557-2562.

[10] Gunstensen, A. K.; Rothman, D. H.; Zaleski, S.; Zanetti, G. Lattice Boltzmann model of immiscible fluids. Physical Review A, 1991, 43, 4320.

[11] Li, W.; Amirfazli, A. Hierarchical structures for natural superhydrophobic surfaces. Soft Matter, 2008, 4, 462-466.

[12] Shi, B.; Guo, Z. Lattice Boltzmann model for nonlinear convection-diffusion equations. Physical Review E, 2009, 79, 016701.

[13] Nakajima, A. Design of hydrophobic surfaces for liquid droplet control. NPG Asia Materials, 2011, 3, 49-56.

[14] Wong, T.-S.; Sun, T.; Feng, L.; Aizenberg, J. Interfacial materials with special wettability. MRS bulletin, 2013, 38, 366-371. 
[15] Joseph, P.; Cottin-Bizonne, C.; Benoit, J.-M.; Ybert, C.; Journet, C.; Tabeling, P.; Bocquet, L. Slippage of water past superhydrophobic carbon nanotube forests in microchannels. Physical Review Letters, 2006, 97, 156104.

[16] Lafuma, A.; Quéré, D. Superhydrophobic states. Nature materials, 2003, 2, 457-460.

[17] Reis, T.; Phillips, T. N. Lattice Boltzmann model for simulating immiscible two-phase flows. Journal of Physics A: Mathematical and Theoretical, 2007, 40, 4033.

[18] Liu, H.; Valocchi, A. J.; Kang, Q. Three-dimensional lattice Boltzmann model for immiscible two-phase flow simulations. Physical Review E, 2012, 85, 046309.

[19] Falcucci, G.; Bella, G.; Shiatti, G.; Chibbaro, S.; Sbragaglia, M.; Succi, S. Lattice Boltzmann models with mid-range interactions. Communications in computational physics, 2007, 2, 1071-1084.

[20] Bormashenko, E. Y.: Wetting of real surfaces; Walter de Gruyter, 2013; Vol. 19.

[21] Li, Q.; Luo, K.; Li, X. Lattice Boltzmann modeling of multiphase flows at large density ratio with an improved pseudopotential model. Physical Review E, 2013, 87, 053301.

[22] Inamuro, T.; Ogata, T.; Tajima, S.; Konishi, N. A lattice Boltzmann method for incompressible two-phase flows with large density differences. Journal of Computational Physics, 2004, 198, 628-644.

[23] Gao, N.; Yan, Y. Modeling Superhydrophobic Contact Angles and Wetting Transition. Journal of Bionic Engineering, 2009, 6, 335-340.

[24] Zu, Y.; Yan, Y. Single Droplet on Micro Square-Post Patterned Surfaces-Theoretical Model and Numerical Simulation. Scientific Reports, 2016, 6.

[25] Gong, W.; Zu, Y.; Chen, S.; Yan, Y. Wetting transition energy curves for a droplet on a square-post patterned surface. Science Bulletin, 2017, 62, 136-142.

[26] Lee, T.; Liu, L. Lattice Boltzmann simulations of micron-scale drop impact on dry surfaces. Journal of Computational Physics, 2010, 229, 8045-8063.

[27] Lee, T.; Lin, C.-L. A stable discretization of the lattice Boltzmann equation for simulation of incompressible two-phase flows at high density ratio. Journal of Computational Physics, 2005, 206, 16-47.

[28] Yan, Y. Y.; Zu, Y. Q.; Dong, B. LBM, a useful tool for mesoscale modelling of singlephase and multiphase flow. Applied Thermal Engineering, 2011, 31, 649-655.

[29] Dupuis, A.; Yeomans, J. Modeling droplets on superhydrophobic surfaces: equilibrium states and transitions. Langmuir, 2005, 21, 2624-2629.

[30] Vrancken, R. J.; Kusumaatmaja, H.; Hermans, K.; Prenen, A. M.; Pierre-Louis, O.; Bastiaansen, C. W.; Broer, D. J. Fully reversible transition from Wenzel to Cassie- Baxter states on corrugated superhydrophobic surfaces. Langmuir, 2009, 26, 3335-3341.

[31] Kusumaatmaja, H.; Yeomans, J. Modeling contact angle hysteresis on chemically patterned and superhydrophobic surfaces. Langmuir, 2007, 23, 6019-6032.

[32] Kusumaatmaja, H.; Vrancken, R.; Bastiaansen, C.; Yeomans, J. Anisotropic drop morphologies on corrugated surfaces. Langmuir, 2008, 24, 7299-7308.

[33] Hyväluoma, J.; Timonen, J. Impact states and energy dissipation in bouncing and non-bouncing droplets. Journal of Statistical Mechanics: Theory and Experiment, 2009, 2009, P06010.

[34] Zhang, J.; Kwok, D. Y. Contact line and contact angle dynamics in superhydrophobic channels. Langmuir, 2006, 22, 4998-5004.

[35] Huang, J. J.; Shu, C.; Chew, Y. T. Lattice Boltzmann study of droplet motion inside a grooved channel. Physics of Fluids (1994-present), 2009, 21, 022103.

[36] Swift, M. R.; Osborn, W.; Yeomans, J. Lattice Boltzmann simulation of nonideal fluids. Physical Review Letters, 1995, 75, 830. 
[37] Pearce, J.; Giustini, A.; Stigliano, R.; Jack Hoopes, P. Magnetic Heating of Nanoparticles: The Importance of Particle Clustering to Achieve Therapeutic Temperatures. Journal of Nanotechnology in Engineering and Medicine, 2013, 4, 110071-1100714.

[38] Yan Y Y, Zu Y Q. A lattice Boltzmann method for incompressible two-phase flows on partial wetting surface with large density ratio. Journal of Computational Physics, 2007, 227(1): 763775.

[39] Briant, A. J.; Wagner, A. J.; Yeomans, J. M. Lattice Boltzmann simulations of contact line motion. I. Liquid-gas systems. Physical Review. E: Statistical, Nonlinear, and Soft Matter Physics, 2004, 69, 031602.

[40] Briant, A. J.; Yeomans, J. M. Lattice Boltzmann simulations of contact line motion. II. Binary fluids. Physical Review. E: Statistical, Nonlinear, and Soft Matter Physics, 2004, 69, 031603.

[41] Inamuro T, Ogata T, Tajima S, et al. A lattice Boltzmann method for incompressible two-phase flows with large density differences. Journal of Computational Physics, 2004, 198(2): 628644.

[42] Zu, Y. Q.; Yan, Y. Y. Lattice Boltzmann method for modelling droplets on chemically heterogeneous and microstructured surfaces with large liquid-gas density ratio. IMA Journal of Applied Mathematics, 2011, 76, 743-760.

[43] Kim, Y. H.; Choi, W.; Lee, J. S. Water droplet properties on periodically structured superhydrophobic surfaces: a lattice Boltzmann approach to multiphase flows with high water/air density ratio. Microfluidics and Nanofluidics, 2010, 10, 173-185.

[44] Tanaka, Y.; Washio, Y.; Yoshino, M.; Hirata, T. Numerical simulation of dynamic behavior of droplet on solid surface by the two-phase lattice Boltzmann method. Computers \& Fluids, 2011, 40, 68-78.

[45] Patankar, N. A. Transition between superhydrophobic states on rough surfaces. Langmuir, 2004, 20, 7097-7102.

[46] Patankar, N. A. Consolidation of hydrophobic transition criteria by using an approximate energy minimization approach. Langmuir, 2010, 26, 8941-8945.

[47] Bormashenko, E. Progress in understanding wetting transitions on rough surfaces. Advances in Colloid and Interface Science, 2015, 222, 92-103.

[48] He, B.; Patankar, N. A.; Lee, J. Multiple equilibrium droplet shapes and design criterion for rough hydrophobic surfaces. Langmuir, 2003, 19, 4999-5003. 